\title{
Effect of Fin Orientation and Forced Convection on the Performance of Metal Foam Fins using a $\mu$-CT Scan Based 3D CFD Model
}

\author{
Khaled S. Al-Athel ${ }^{1, a, *}$ and Moayed Al-Harbi ${ }^{1, b}$ \\ ${ }^{1}$ Mechanical Engineering Department, King Fahd University of Petroleum \& Minerals, Dhahran, \\ Saudi Arabia 31261 \\ a.kathel@kfupm.edu.sa,b.s201171830@kfupm.edu.sa \\ *corresponding author \\ Tel. \#+966-13-8608831 \\ Fax \# +966-13-8602949
}

Keywords: Metal Foam, Fin, micro-CT scan, CFD, Convective Heat Transfer.

\begin{abstract}
Metal foams are very attractive materials for thermal and electronic packaging applications due to their improved heat transfer capabilities. Their improved heat transfer effective properties are due to the relatively large contact area they possess because of their cell structure. This comes at the expense of the pressure drop. This work presents a methodology based on $\mu$-CT scan to develop a realistic metal foam 3D model. The model is validated with experiments and used to study the behavior of the metal foam as a fin in terms of the temperature variation within the fin as well as the effect of the airflow velocity and fin orientation to the pressure drop. Results showed two major observations. First, this methodology could be used to identify a velocity value at which the fin orientation becomes obsolete and has no effect on the temperature variation. Second, the pressure drop alone could not be used to assess the fin, but also the fin orientation has to be taken into account to examine the total pressure drop.
\end{abstract}

\section{Introduction}

Open cell metal foams have been getting more attention recently in heat transfer and heat recovery applications. Due to their geometrical nature, the open cells increase the surface area in contact with any cooling fluid, leading to a more rapid temperature drop in any electrical or mechanical component. Two major drawbacks used to hinder the use of metallic foams, their manufacturability, and the pressure drop associate with their use. Recent advances in 3D printing, casting, and other techniques have helped in overcoming any issues related to manufacturing the desired foam shape for an application. The problem with the pressure drop cannot be avoided nor solved, but a more detailed analysis and understanding is needed to identify if the amount of pressure drop is considered relatively high or not. 
In addition to their large surface area, metal foams have higher effective heat transfer properties because of the randomness in the cell structure. Therefore, it is sometimes difficult to identify a best configuration for a metal foam in a thermal or electronic packaging application without testing the model first. Experiments are useful but mostly for effective properties rather than a detailed assessment of the metal foam component. Therefore, the use of computational means has been a lot in the literature to assess the mechanical and thermal behavior of metallic metal foams [1-4].

A common approach in the literature is to model the metal foam using its effective properties without the need to have the actual geometrical representation. This approach overcomes the issues that comes with the difficulty in having a realistic 3D model but gives results in terms of the effective performance instead of a detailed analysis. Odabaee and Hooman [5] studied the effect of various parameters such as free stream velocity, metal foam thickness, metal foam porosity, etc. on the heat and fluid flow in metal foam heat exchangers. The numerical model used in their work modeled the metal foam as an isotropic homogenous porous media. Bayomy et al. [3, 6] used effective metal foam properties to simulate the metal foam behavior and investigate the heat transfer characteristics in a metal foam heat sink. The approach is useful to study the behavior of the metal foam based heat sink design, but one has to pay attention to the calculation of the effective properties, as the accuracy of the model will depend on it.

A more involved approach is to develop geometrical construction of the metal foam open cells. This approach would allow a more detailed analysis of the fluid flow within the foam and would give results that are more detailed. Kopanidis et al. [1] constructed a 3D geometrical model for few metal foam open cells. The cells were used to generate a larger foam sample using symmetry. Finally, a random part of the large foam sample was cut out to produce a more random metal foam cell structure. The model was used to study the fluid flow characteristics of metal foams. A similar approach was used by Peng et al. [7] by using a body centred structure to construct a single cell. The unit cell model was used to compute the effective thermal conductivity and analyse the heat transfer at the gas-solid interface. This approach of the 3D geometrical construction is based on the Lord Kelvin's model and is not practical for performance analysis of thermal applications.

The most appropriate approach for detailed analysis of metal foams and metal foam components is the use of $\mu$-CT scan to develop a realistic 3D representation. Della Torre et al. [8] used $\mu$-CT scan to study the micro-structure of the foam. Mathematical calculations based on the CT scan model were compared with numerical computations of an ideal geometry. The models were used to study the fluid flow and the pressure drop. Ranut et al. $[2,9]$ used $\mu$-CT scan to develop a 3D metal foam geometry that was used to study the flow and thermal behavior using CFD analysis at the pore level. A detailed analysis on the effect of fluid flow Reynolds number on the heat transfer coefficient was presented. The model was useful in providing detailed analysis of the metal foam and its thermal behavior.

A similar $\mu$-CT scan model was developed by Al-Athel et al. $[4,10]$ and used to study the thermo-mechanical behavior of metal foam heat sinks. The model used was validated against experiments and used to calculate the heat transfer coefficient under free and forced convection. It is of importance to note that the foam geometry used was bigger than a unit cell, which is required to capture the number of pores per inch (PPI). This has an advantage of being able to look at the cell level to extract any needed data, in addition to studying the thermal behavior of the heat sink component as a whole.

In this work, the metal foam model developed in $[4,10]$ is used to study the temperature variations and pressure drop in metal foam fins using computational fluid dynamics (CFD) analysis. In the literature, there exists a lot of work on metal foams using CFD and most of it can be 
categorized into two types: Using an effective model but on a large scale or using a $\mu$-CT scan model but on a unit cell model. This work utilized a heat sink $\mu$-CT scan model fin-size model using CFD analysis.

\section{Metal Foam $\mu$-CT Scan Model}

To develop a realistic 3D metal foam model, $\mu$-CT scan is used on a metal foam sample of size $25 \times 25 \times 6.35 \mathrm{~mm}$ made of 6101 aluminum alloy from GoodFellow [11]. The sample has pore density of 4PPC (pores-per-centimetres, i.e. $10 \mathrm{PPI}$ ) and porosity of 93\%. The planar dimensions of the sample $(2.5 \times 2.5 \mathrm{~cm})$ cover the minimum dimensions required to capture the foam pore density of PPC. The metal foam model was developed through three stages. First, Skyscan 1172 was used to scan the model using an X-ray with electron gun voltage of $40 \mathrm{kV}$ and gun current of $250 \mu \mathrm{A} .512$ slice images were captured using a rotational step size of $0.7^{\circ}$. The resulted image pixel size was $27.34 \mu \mathrm{m}$. The 3D model can then be imported into any CAD software to clean it and generate a 3D surface model. This model represents the geometry of the foam but is hollow from the inside and can only have a surface mesh. In the final step, the 3D surface model is imported into any FE or CFD software to generate the 3D solid model that can be used for any heat transfer or CFD analysis. Figure 1 summarizes the steps for developing the 3D $\mu$-CT scan model. More details on the scanning parameters can be found in [10].

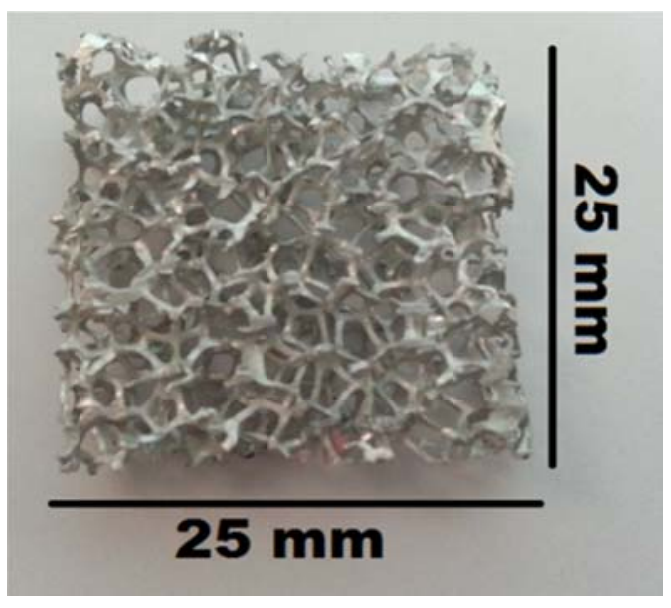

(a) Metal foam sample

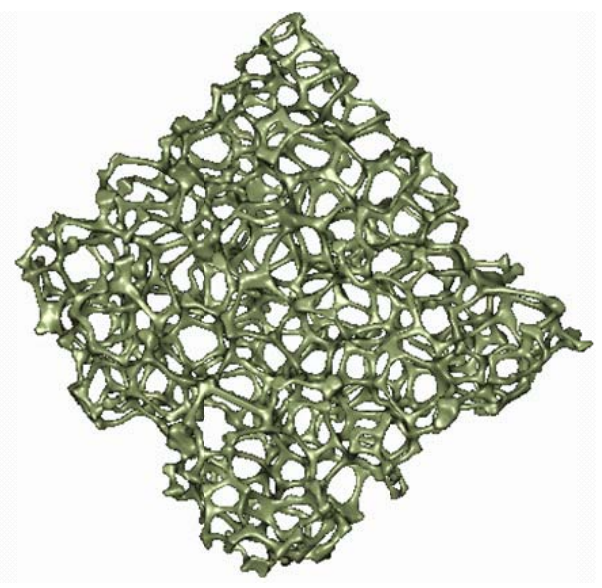

(c) Cleaned CAD surface model

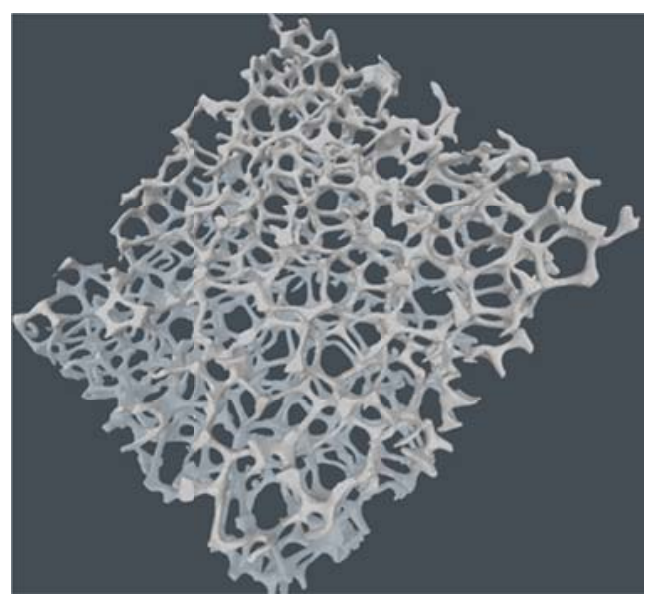

(b) Final CT scan model

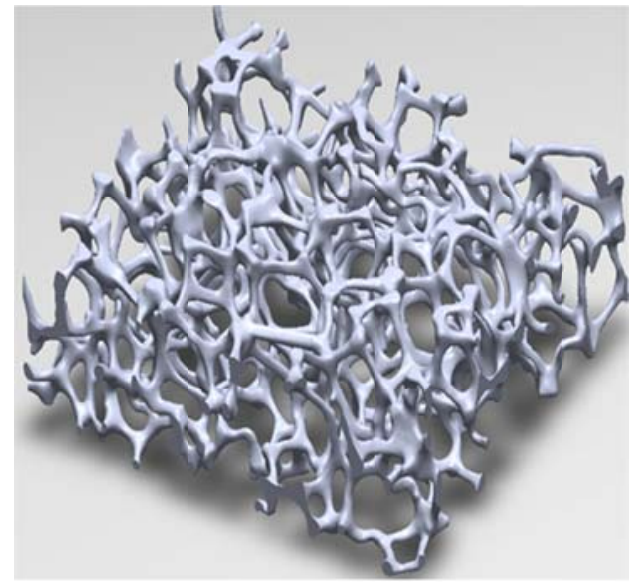

(d) Final 3D volume model

Figure 1 Metal Foam 3D Modelling Steps. 


\section{Metal Foam CFD Model Validation}

Before the use of the model in the CFD analysis, the 3D model developed from the $\mu$-CT scan approach has to be validated. In this case, an experimental setup is used and the results are compared with the computational values. The metal foam sample is attached to a heat source with heat input of $5 \mathrm{~W}$. The metal foam was heated until the heat source and the bottom of the foam reached $60^{\circ} \mathrm{C}$, at which, a cooling fan is turned on with air velocity of $3.6 \mathrm{~m} / \mathrm{s}$, while adapting the heat source to keep the temperature at $60^{\circ} \mathrm{C}$. The temperatures were recorded until the foam reaches steady state. The airflow was perpendicular to the metal foam $\left(90^{\circ}\right)$ and thermocouples were attached at various locations to measure the temperature change with time. The details of the experimental setup are shown in Figure 2.

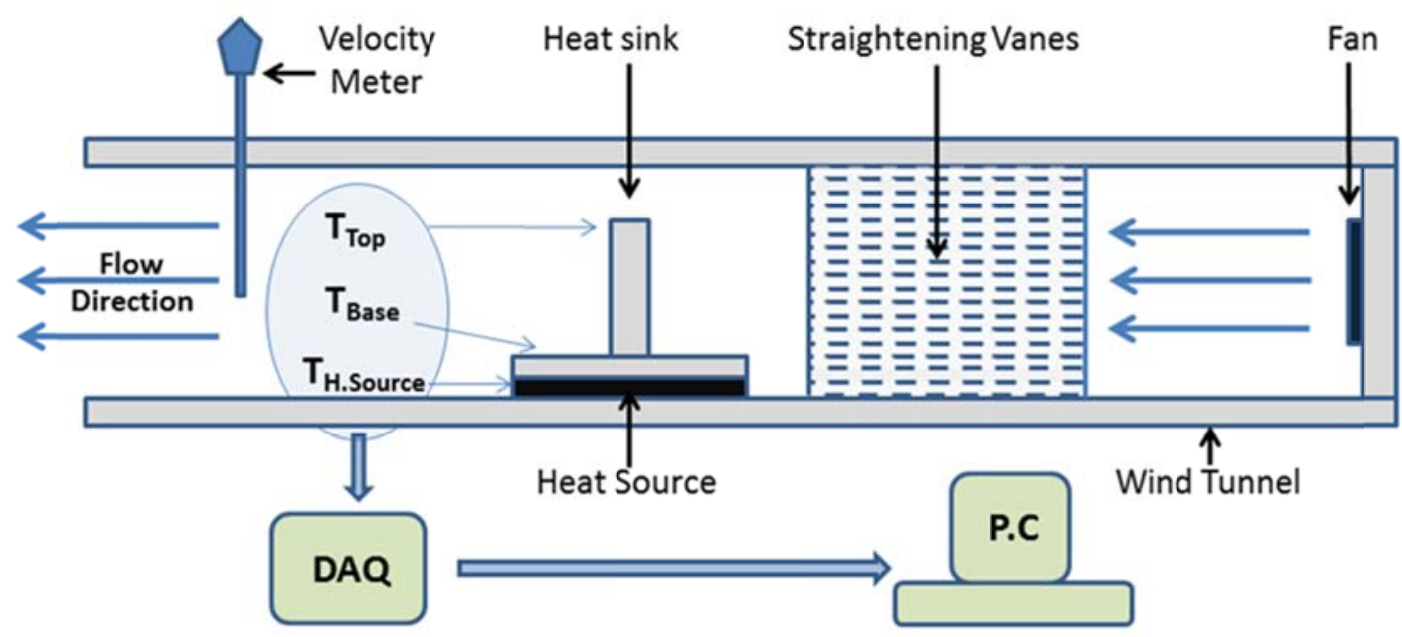

Figure 2 Schematic of Metal Foam Forced Convection Experimental Setup.

For the CFD simulations, the metal foam was placed in a computational domain large enough to allow the air streams to travel through and around the model and reach steady state. The metal foam fin was set to be at room temperature of $25^{\circ} \mathrm{C}$. The bottom of the metal foam was set to reach $60^{\circ} \mathrm{C}$ in $15 \mathrm{~min}$ (experiment took around 15 minutes for the base to reach $60^{\circ} \mathrm{C}$ ), and then the air was given a velocity of $3.6 \mathrm{~m} / \mathrm{s}$. Because of the cooling fan, the air was given an initial temperature of $22^{\circ} \mathrm{C}$ as measured from experiment. Figure 3 shows the setup of the CFD simulation.

The temperature variation with time from the experiment and the CFD simulation is shown in Figure 4. As can be seen in the figure, the 3D CFD model predicts the temperature variation very closely to the experimental results. It is important to note that the exact matching in the base temperature between the two curves is due to the fact that the $60^{\circ} \mathrm{C}$ was enforced in both the experiment as well as the simulation. At this point, the CFD 3D $\mu$-CT scan model of the metal foam fin can be used with confidence to perform the desired parametric study. 


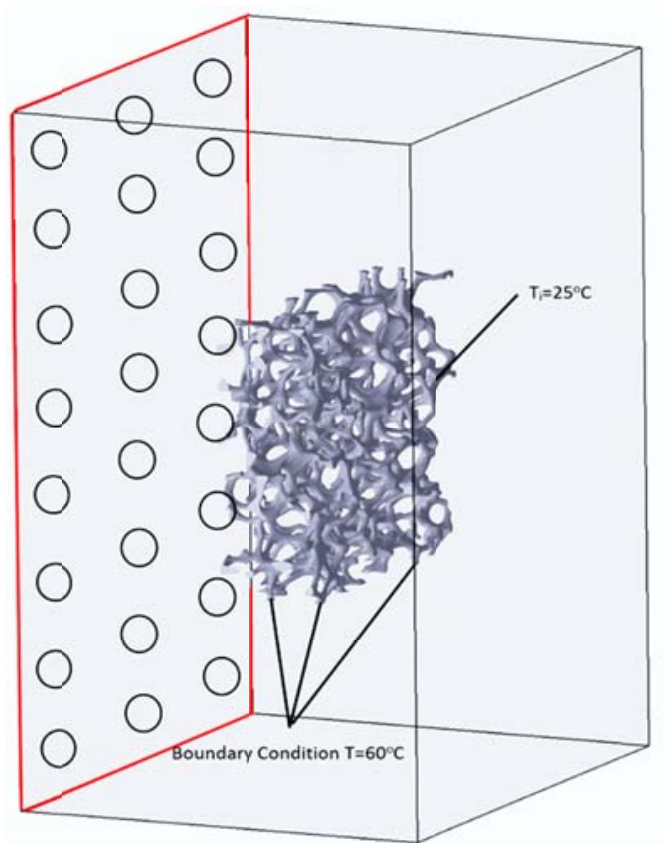

Figure 3 CFD Boundary and initial conditions.

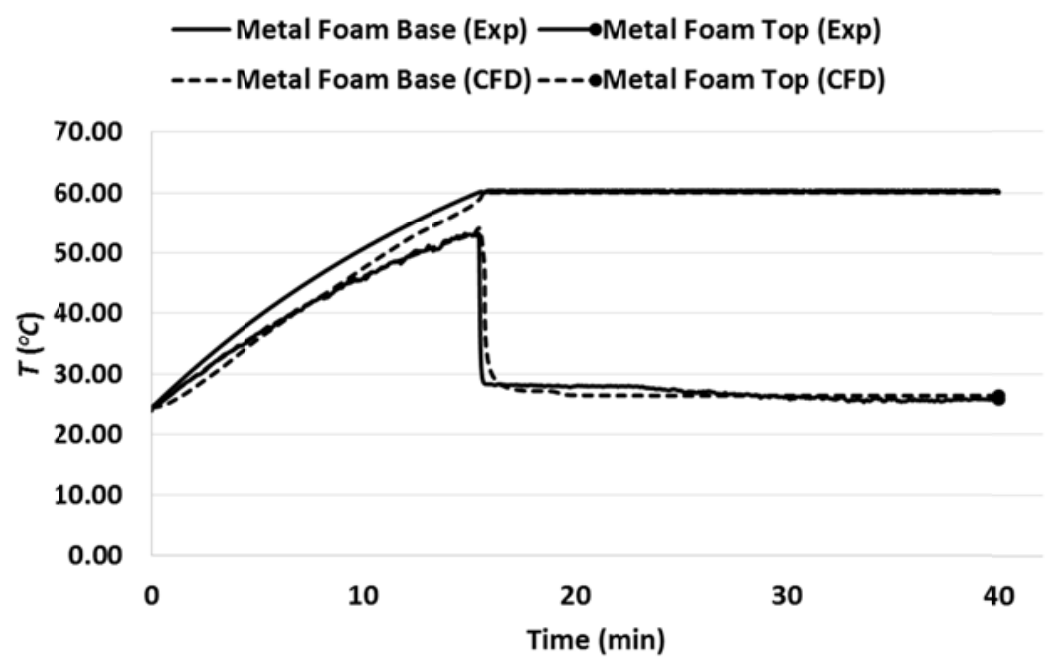

Figure 4 Temperature variation at the base and top of the metal foam.

\section{Metal Foam CFD Analysis}

This study focuses on the effect of two parameters on the metal foam fin performance, the orientation and the forced convection air velocity. The set of operating conditions is the same as the conditions used for the validation. The set of simulations is listed in Table 1, and the material properties for 6101 aluminum alloy are listed in Table 2.

For each CFD analysis, the temperature distribution is captured at steady state in addition to the air velocity streamlines for analysis of the flow behavior. Figure 5Figure 7 show the temperature distribution and the airflow streamlines for the nine cases. 
Table 1 Metal foam fin CFD set of simulations.

\begin{tabular}{ccc} 
Case & Orientation & Air Velocity \\
\hline 1 & $90^{\circ}$ & \\
2 & $45^{\circ}$ & $1 \mathrm{~m} / \mathrm{s}$ \\
3 & $0^{\circ}$ & \\
\hline 4 & $90^{\circ}$ & \\
5 & $45^{\circ}$ & $5 \mathrm{~m} / \mathrm{s}$ \\
6 & $0^{\circ}$ & \\
\hline 7 & $90^{\circ}$ & \\
8 & $45^{\circ}$ & $10 \mathrm{~m} / \mathrm{s}$ \\
9 & $0^{\circ}$ & \\
\hline
\end{tabular}

Table 2 Material properties of 6101 aluminum alloy.

\begin{tabular}{cc} 
Property & Value \\
\hline Density $\left(\mathrm{Kg} / \mathrm{m}^{3}\right)$ & 2700 \\
Thermal Conductivity $(\mathrm{W} / \mathrm{mK})$ & 218 \\
Coefficient of thermal Expansion $\left(1 /{ }^{\circ} \mathrm{C}\right)$ & $23.58 \times 10^{-6}$ \\
Specific heat $(\mathrm{J} / \mathrm{Kg} . \mathrm{K})$ & 895
\end{tabular}

A major advantage of temperature distribution results in Figure 5Figure 7 is that the CT scan model is detailed and can be used to extract the special temperature distribution at any point instead of having average values in effective models. Another observation can be clearly seen by comparing the temperature field in Figure 5,Figure 6, and Figure 7. In the case of airflow with $90^{\circ}$ fin orientation, the temperature values are almost uniform along every cross section, whereas for $0^{\circ}$, and to some degree at $45^{\circ}$, there is a special temperature gradient at both horizontal and vertical axes. These two observations provided by the $\mu$-CT scan model can be very useful for designers of heat sinks, heat exchangers, and other applications that require the use of a heat transfer cooling system. Two major design parameters could be looked at for example, the efficiency or effectiveness of the fin, and the thermal resistance. The effectiveness of a fin in a heat sink could be defined as [12]:

$$
\text { Effectiveness }=R_{C} \frac{\left(T_{b}-T_{f i n}\right)}{\left(T_{b}-T_{a m b}\right)}
$$

where $R_{c}$ is the heat sink capacity, $T_{b}$ is the base temperature, $T_{f i n}$ is the fin temperature at the top, and $T_{a m b}$ is the ambient temperature. The thermal resistance is defined as [13]:

$$
R_{t h}=\frac{\left(T_{b}-T_{a m b}\right)}{Q}
$$

where $Q$ is the total dissipated power. As can be seen from Eq. (1) and (2), the temperature data is important in calculating such parameters. The advantage of having a detailed model is the ability to utilize the temperature field data available to optimize the size and shape of the fin to minimize the thermal resistance and increase the efficiency. The analysis provided here does not require the 
computation of the heat transfer coefficient $h$ as in previous studies [4]. The airflow streamlines provides insight on the disturbance of the airflow because of the metal foam nature. From the analysis, it can be seen that fin orientation of $45^{\circ}$ is worst when it comes to the disturbance, whereas the $90^{\circ}$ gives the least drop in the airflow velocity.
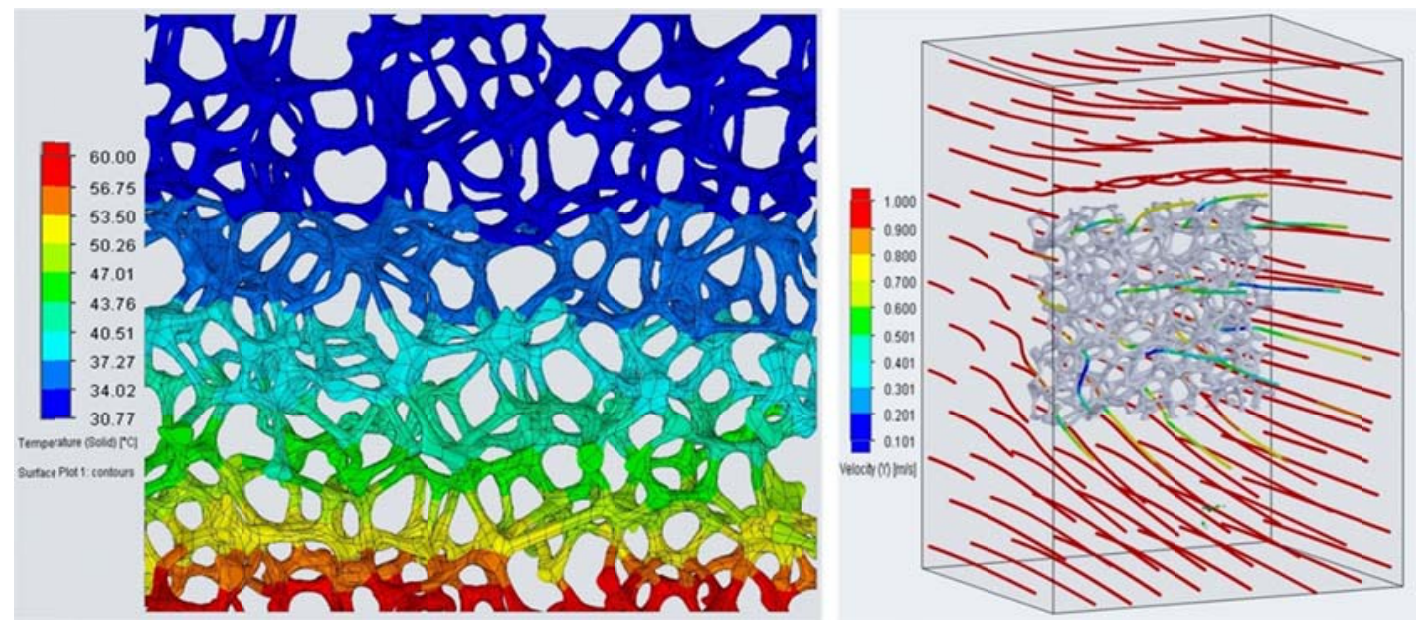

(a) Airflow velocity of $1 \mathrm{~m} / \mathrm{s}$
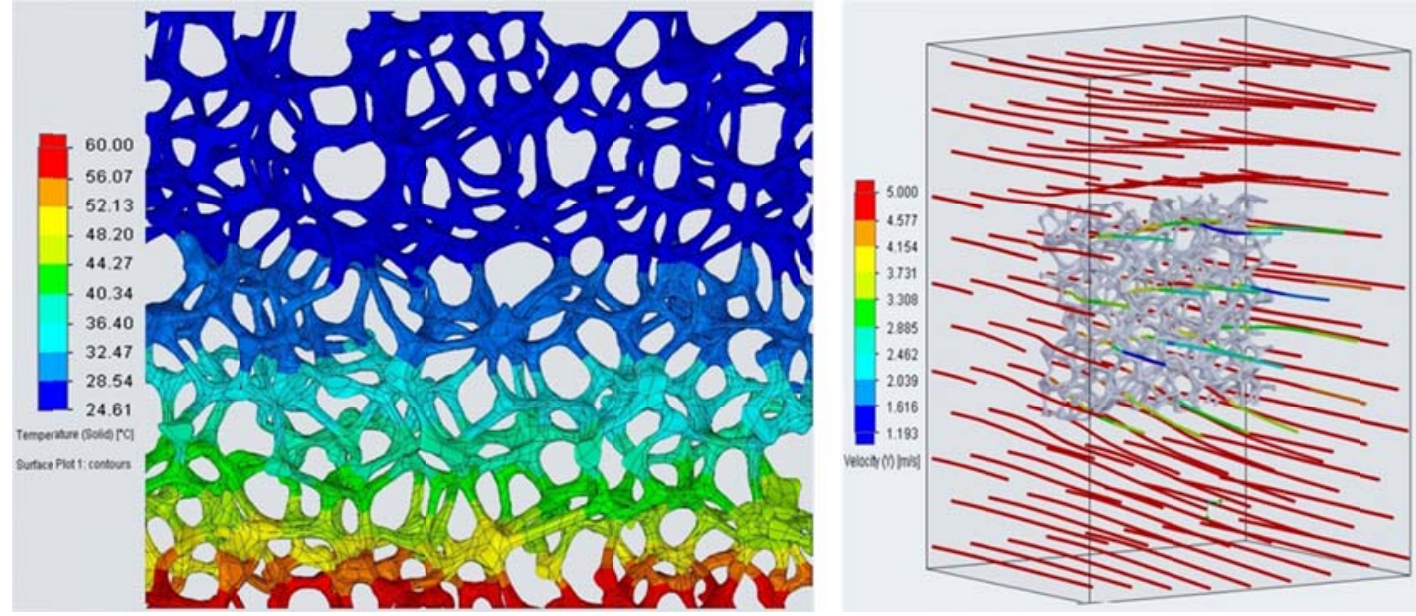

(b) Airflow velocity of $5 \mathrm{~m} / \mathrm{s}$
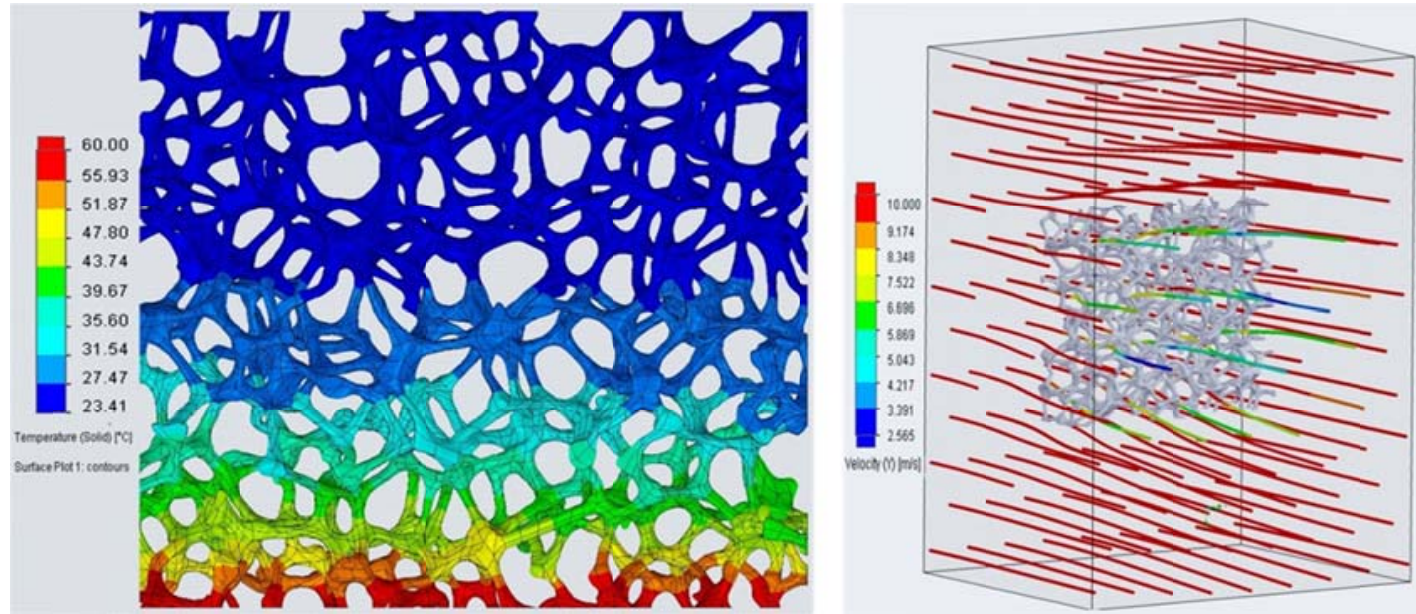

(c) Airflow velocity of $10 \mathrm{~m} / \mathrm{s}$

Figure 5 Temperature distribution and airflow streamlines for $90^{\circ}$ Orientation. 

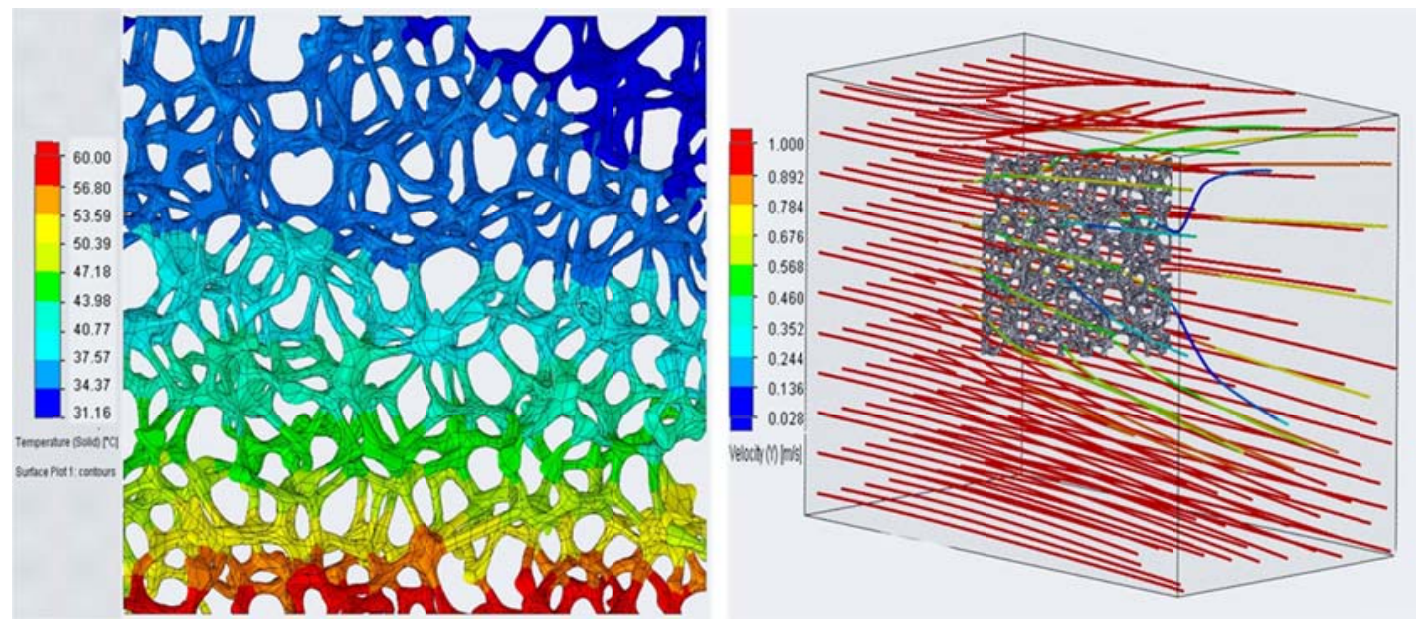

(a) Airflow velocity of $1 \mathrm{~m} / \mathrm{s}$

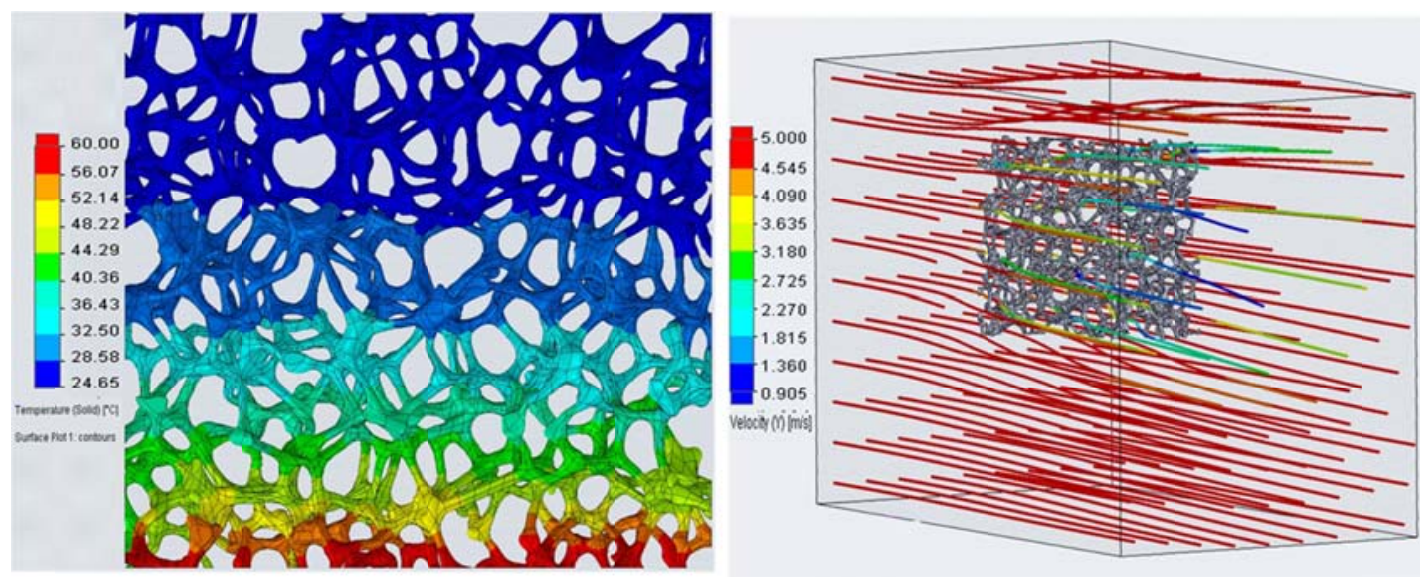

(b) Airflow velocity of $5 \mathrm{~m} / \mathrm{s}$

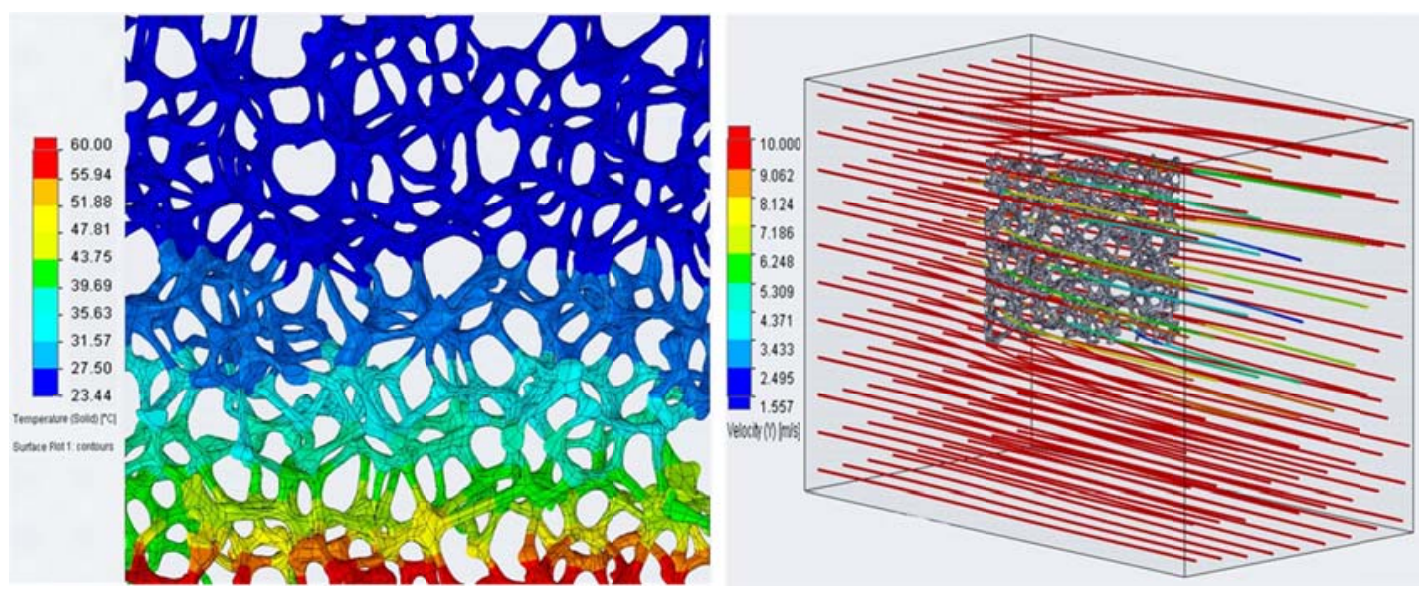

(c) Airflow velocity of $10 \mathrm{~m} / \mathrm{s}$

Figure 6 Temperature distribution and airflow streamlines for $45^{\circ}$ Orientation. 

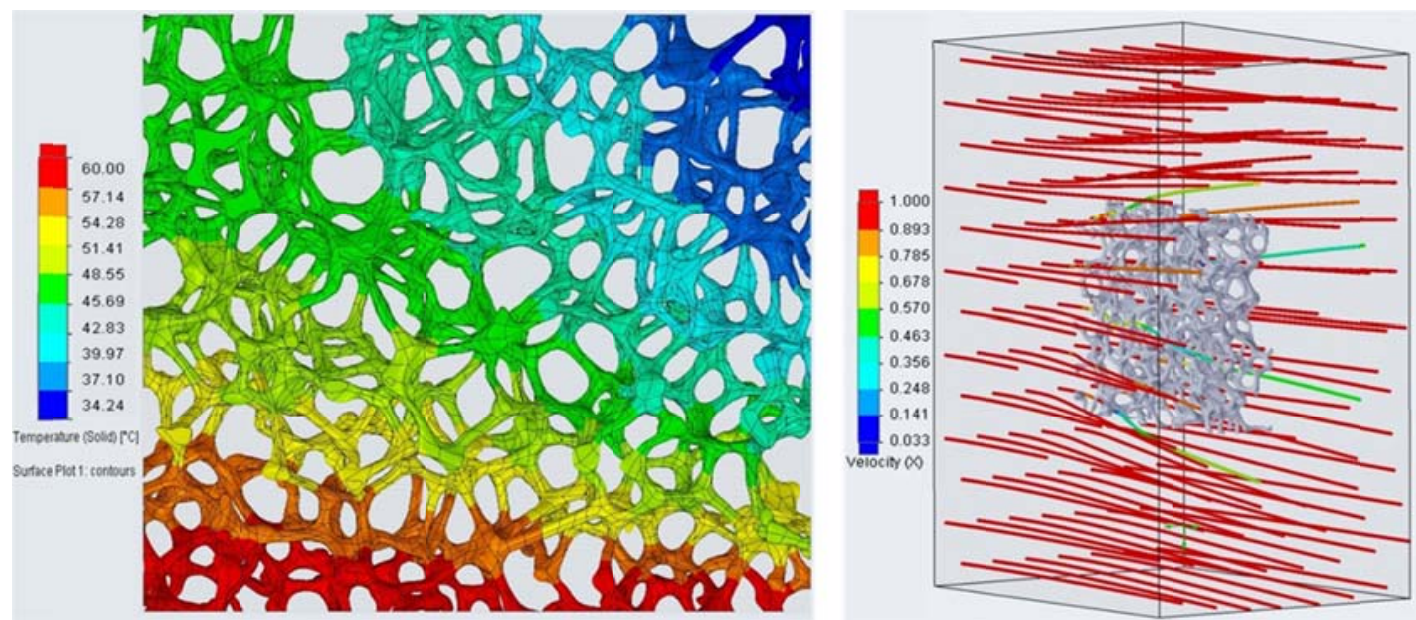

(a) Airflow velocity of $1 \mathrm{~m} / \mathrm{s}$
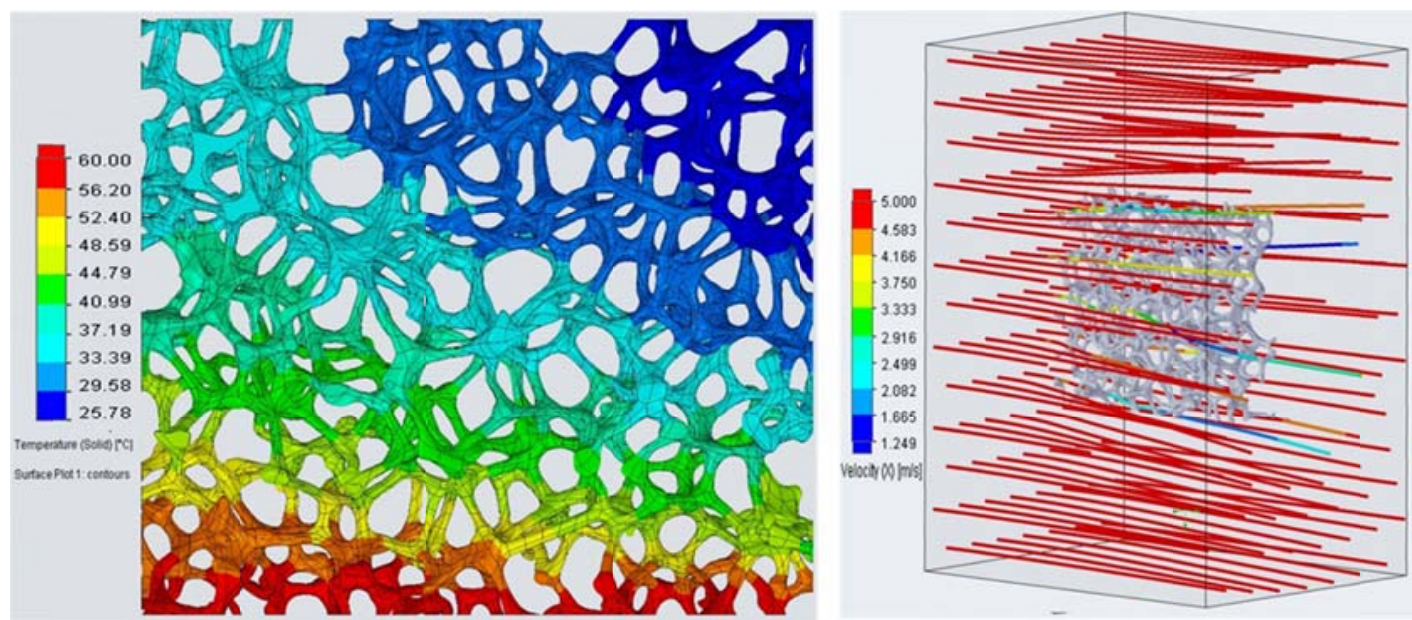

(b) Airflow velocity of $5 \mathrm{~m} / \mathrm{s}$
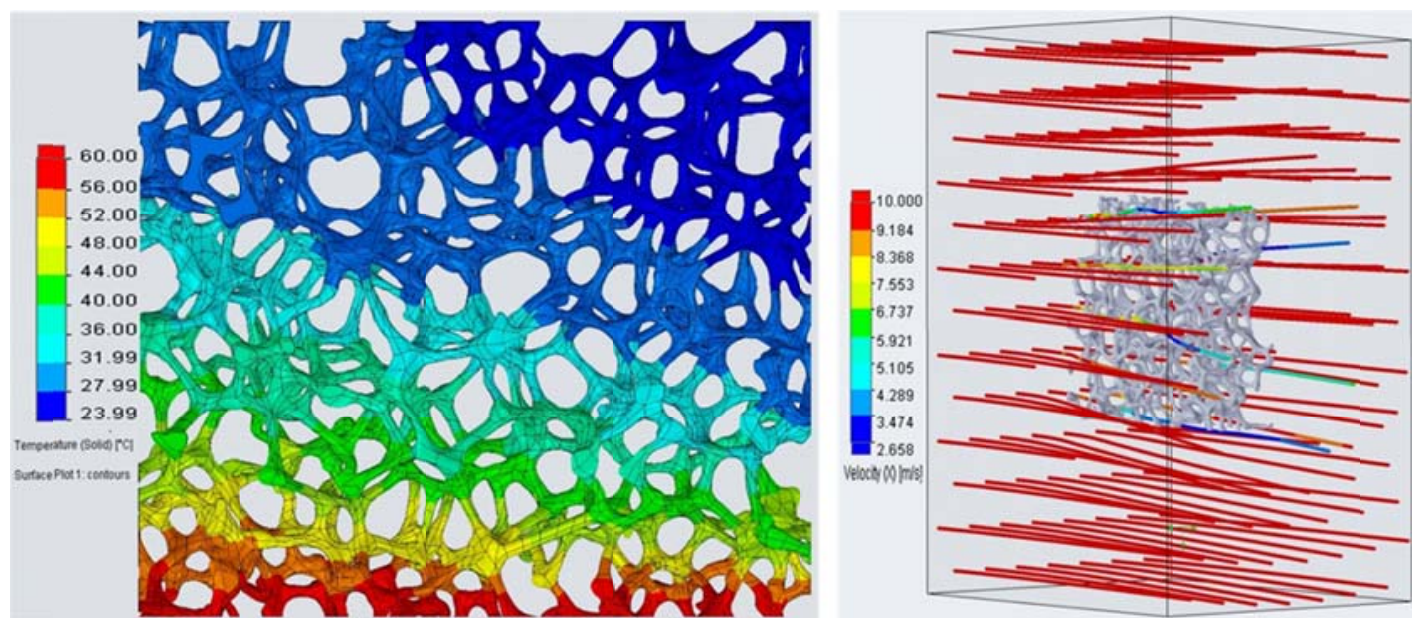

(c) Airflow velocity of $10 \mathrm{~m} / \mathrm{s}$

Figure 7 Temperature distribution and airflow streamlines for $0^{\circ}$ Orientation. 


\section{Results and Discussion}

\subsection{Temperature Variation}

The metal foam fin in a heat sink application is supposed to dissipate heat via convection. First, we look into the maximum temperature variation within the metal foam fin as a function of airflow velocity. Figure 8 shows the temperature variation for all three orientations as a function of the airflow velocity. All fin orientations seems to have the same trend with the airflow velocity when it comes to the maximum temperature difference. At a certain velocity, the temperature difference becomes constant and does not increase with increasing airflow velocity. More importantly, from a design point view, the point at which this occurs appears to be the same for all the cases regardless of the fin orientation.

Figure 9 shows the maximum temperature variation as a function of the fin orientation. Similar to Figure 8 , the fin orientation does not have much effect on the temperature drop for $0^{\circ}$ and $45^{\circ}$ orientations, whereas the $90^{\circ}$ has a significant impact, especially for low airflow velocities. At higher airflow velocities, 5 and $10 \mathrm{~m} / \mathrm{s}$, the fin orientation has no effect on the maximum temperature drop within the metal foam fin. It is important to note that these results and observations are not to be taken as absolute rules, as they only compare the maximum variation but not the distribution. For example, cases with $0^{\circ}$ orientation have temperature variation along both the horizontal and vertical axes as shown in Figure 7.

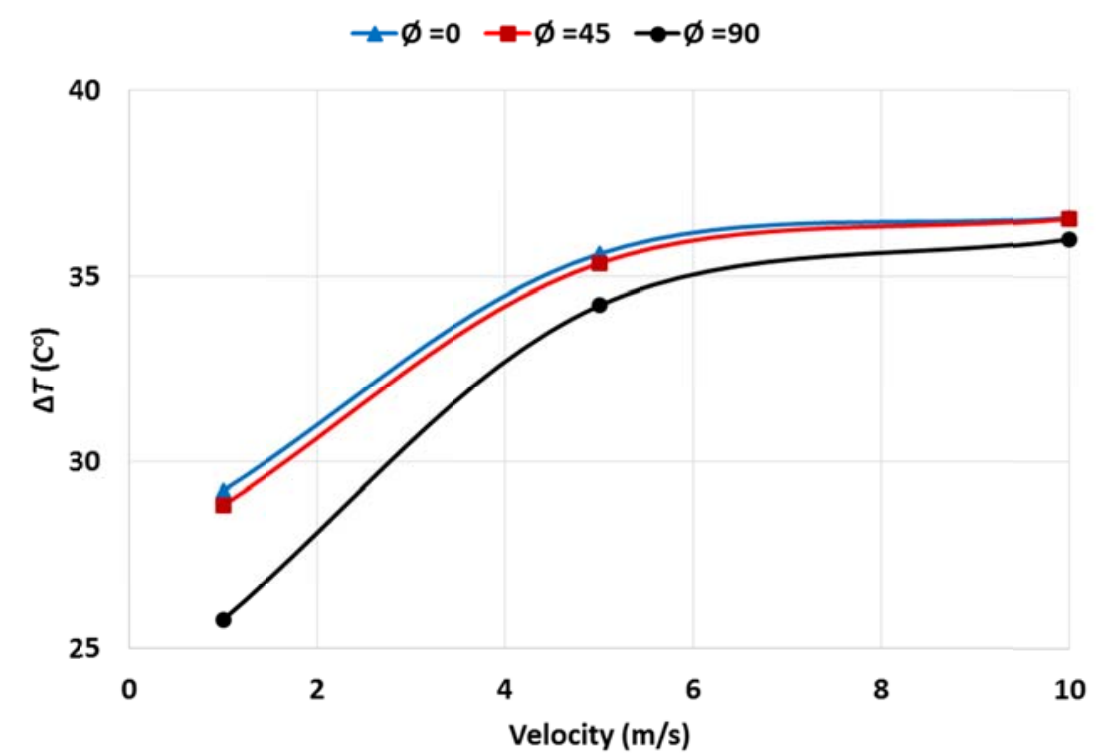

Figure 8 Metal foam fin maximum temperature variation as a function of airflow velocity. 


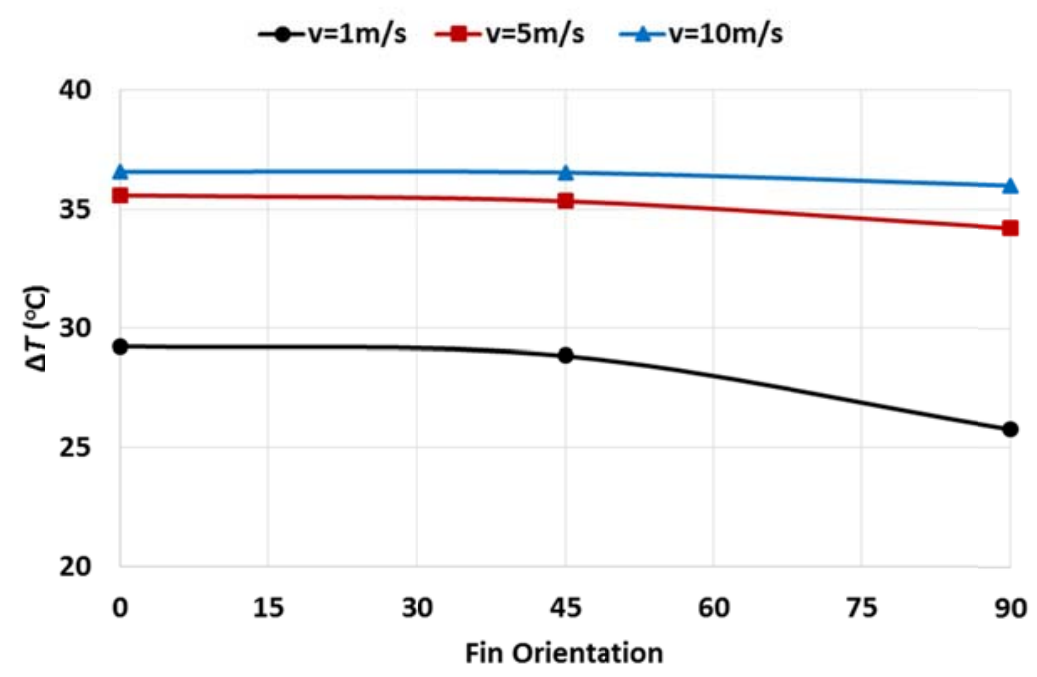

Figure 9 Metal foam fin maximum temperature variation as a function of fin orientation.

\subsection{Pressure Drop}

Another important factor to investigate in thermal applications using metal foams is the pressure drop. Usually, due to the random nature of the foam pores, a significant pressure drop occurs. Depending on the nature of the application, this drop might be acceptable to a certain degree. The airflow pressure can be calculated as:

$$
P=\frac{1}{2} \rho v^{2}
$$

where $\rho$ is the density and $v$ is the airflow velocity. Figure 10 and Figure 11 show the airflow pressure drop per unit length as a function of the airflow velocity and fin orientation, respectively. For very low velocities, the pressure drop is insignificant as the metal foam fin is close to behaving in its natural convection state. That being said, there is some effect of the fin orientation as seen in Figure 10. It is important to note that even though a fin orientation of $0^{\circ}$ appears to have the lowest pressure drop per unit length for low-intermediate velocities, its orientation allows for more total pressure drop, as its total length is larger than the others are.

The behavior changes as the airflow velocity increases, as the $0^{\circ}$ and $45^{\circ}$ becomes closer and $90^{\circ}$ becomes the base with the least pressure drop per unit length. The only significant decrease in pressure drop for high velocities occurs at $90^{\circ}$ orientation. In this case, the velocity is considered high enough to overcome the disturbance of the foam geometry and minimize the pressure drop per unit length. The $45^{\circ}$ orientation has the maximum pressure drop per unit length in all the cases. The reason behind this is that parts of the streamlines of the air disturbed by the foam geometry gets in the way of the remaining streamlines that has not been in contact with foam yet. In the case of $0^{\circ}$ and $90^{\circ}$ orientations, any disturbed streamlines are reflected to the sides of the foam. Therefore, a designer would not only look into the effect of airflow velocity on the pressure drop per unit length, but also to the effect of the fin orientation. 


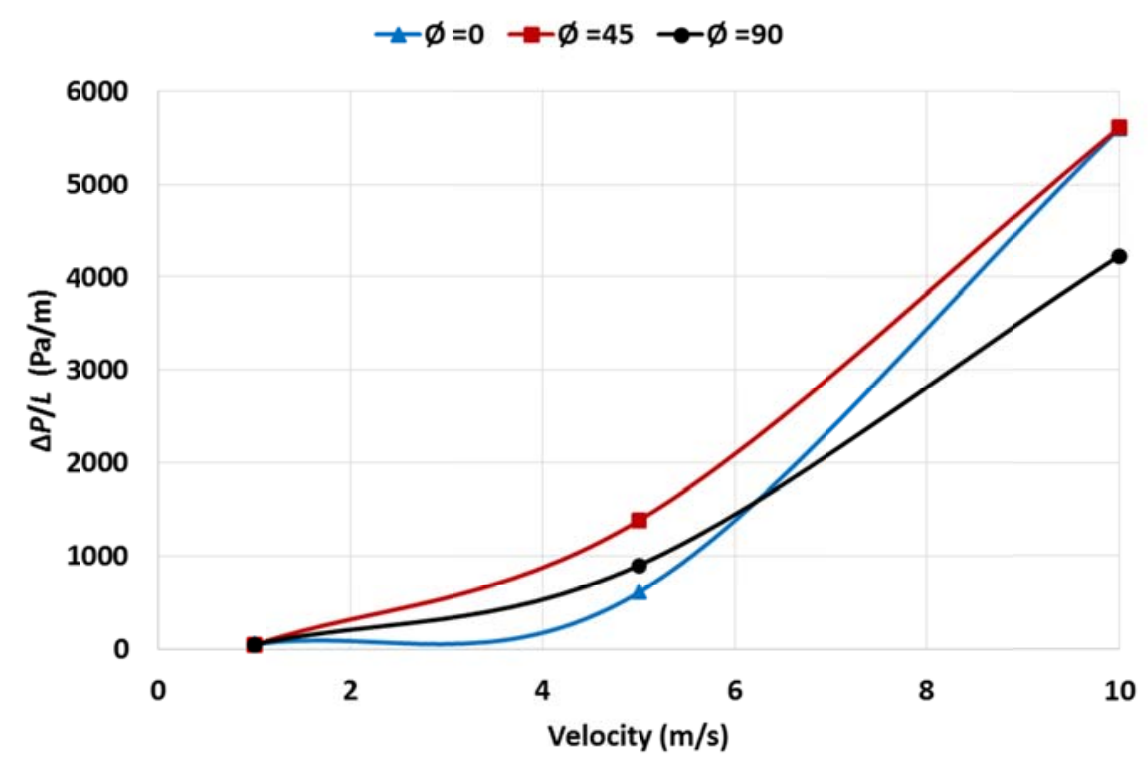

Figure 10 Pressure drop as a function of airflow velocity.

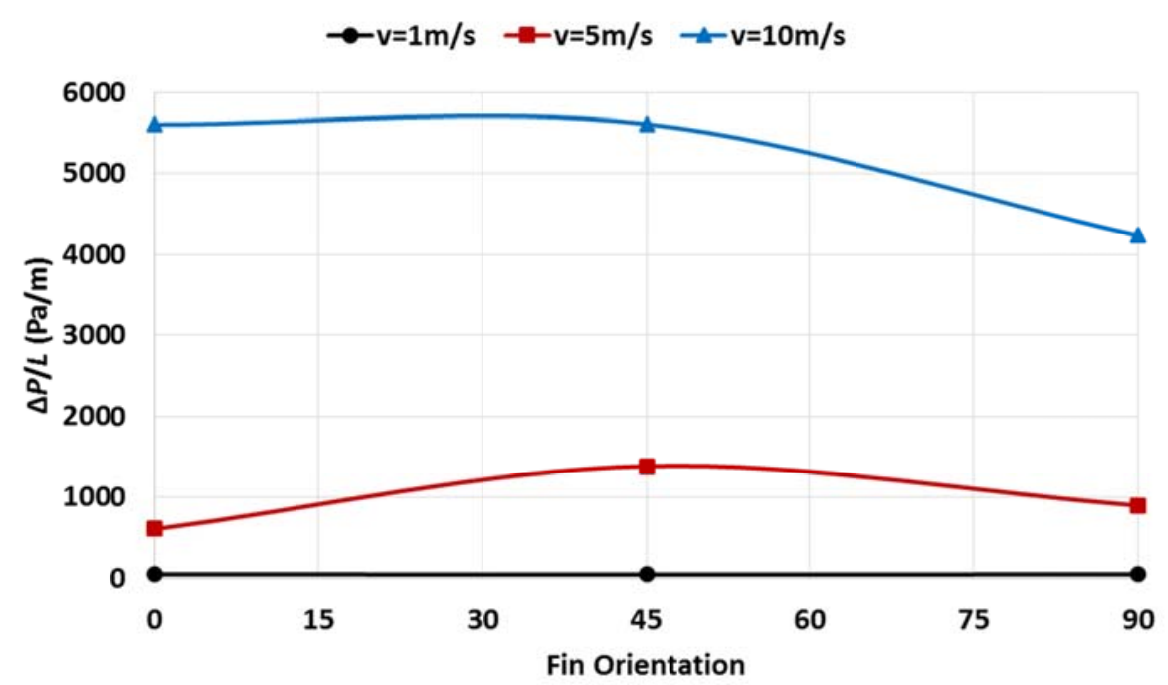

Figure 11 Pressure drop as a function of the fin orientation.

\section{Conclusions}

This study provided a complete $\mu$-CT scan based methodology to assess the behavior of metal foam fins that could be used in thermal and electronic packaging applications using CFD. The model was previously presented $[4,10]$ to assess the thermos-mechanical behavior of metal foam heat sinks using thermal analysis. The model was extended in this study for a CFD analysis to investigate the special temperature variation and the pressure drop.

A combination of three airflow velocities and three metal foam fin orientations (with respect to the airflow) were studies. The effect of fin orientation on the horizontal and vertical temperature distribution was presented. In addition, the effect on pressure drop was quantified per unit length for the same cases. Having a fin orientation of $0^{\circ}$ (parallel to the airflow) causes the temperature field to vary not only on the vertical axis, but also for the horizontal axis. This has a great impact as it 
means that efficiency of the metal foam fin is not the same for the whole fin. This could lead to more optimized designs of the fin cross section that would give the fin a uniform efficiency.

The analysis also showed that, depending on the size of the metal foam fin, the effect of the fin orientation on the temperature variation within the fin becomes negligible at high velocities. Actually, this appears to happen at a fixed velocity value for all cases.

The pressure drop was calculated per unit length and analyzed as a function of the airflow velocity and fin orientation. It is of high importance to not only look at the pressure drop values, but to examine the fin orientation associated with it. For example, a fin orientation of $0^{\circ}$ appears to have the least pressure drop per unit length for low to intermediate velocities. That being said, the $0^{\circ}$ orientation is the case where the airflow has to pass through more metal foam as compared with $45^{\circ}$ and $90^{\circ} \mathrm{m}$ therefore making it the worst case for total pressure drop.

The methodology provided in this study extends on previous work presented in the literature that utilized $\mu$-CT scan to develop a realistic metal foam model. The model presented in this work is of relatively large size and has $2.5 \mathrm{x}$ the minimum length required to capture the real behavior.

\section{Acknowledgements}

The author would like to acknowledge the support of King Fahd University of Petroleum and Minerals through DSR project \# SB131002.

\section{References}

[1] A. Kopanidis, A. Theodorakakos, E. Gavaises, and D. Bouris, "3D numerical simulation of flow and conjugate heat transfer through a pore scale model of high porosity open cell metal foam," International Journal of Heat and Mass Transfer, vol. 53, pp. 2539-2550, May 2010.

[2] P. Ranut, E. Nobile, and L. Mancini, "High resolution microtomography-based CFD simulation of flow and heat transfer in aluminum metal foams," Applied Thermal Engineering, vol. 69, pp. 230-240, Aug 2014.

[3] A. M. Bayomy and M. Z. Saghir, "Heat transfer characteristics of aluminum metal foam subjected to a pulsating/steady water flow: Experimental and numerical approach," International Journal of Heat and Mass Transfer, vol. 97, pp. 318-336, Jun 2016.

[4] K. S. Al-Athel, "A computational methodology for assessing the thermal behavior of metal foam heat sinks," Applied Thermal Engineering, vol. 111, pp. 884-893, Jan 252017.

[5] M. Odabaee and K. Hooman, "Metal foam heat exchangers for heat transfer augmentation from a tube bank," Applied Thermal Engineering, vol. 36, pp. 456-463, Apr 2012.

[6] A. M. Bayomy, M. Z. Saghir, and T. Yousefi, "Electronic cooling using water flow in aluminum metal foam heat sink: Experimental and numerical approach," International Journal of Thermal Sciences, vol. 109, pp. 182-200, Nov 2016.

[7] W. P. Peng, M. Xu, X. F. Li, X. L. Huai, Z. G. Liu, and H. S. Wang, "CFD study on thermal transport in opencell metal foams with and without a washcoat: Effective thermal conductivity and gas-solid interfacial heat transfer," Chemical Engineering Science, vol. 161, pp. 92-108, Apr 62017.

[8] A. Della Torre, G. Montenegro, G. R. Tabor, and M. L. Wears, "CFD characterization of flow regimes inside open cell foam substrates," International Journal of Heat and Fluid Flow, vol. 50, pp. 72-82, Dec 2014.

[9] P. Ranut, E. Nobile, and L. Mancini, "High resolution X-ray microtomography-based CFD simulation for the characterization of flow permeability and effective thermal conductivity of aluminum metal foams," Experimental Thermal and Fluid Science, vol. 67, pp. 30-36, Oct 2015.

[10] K. S. Al-Athel, S. P. Aly, A. F. M. Arif, and J. Mostaghimi, "3D modeling and analysis of the thermomechanical behavior of metal foam heat sinks," International Journal of Thermal Sciences, vol. 116, pp. 119$213,2017$.

[11] GoodFellow. Available: http://www.goodfellow.com/

[12] J. N. J. Deans , W. Dempster, C. K. Lee, "The Use of Effectiveness Concepts to Calculate the Thermal Resistance of Parallel Plate Heat Sinks," Heat Transfer Engineering, vol. 27, pp. 56-67, 2006. 
[13] S. P. Aly, A. F. M. Arif, K. S. Al-Athel, J. Mostaghimi, and S. M. Zubair, "Performance of open pore metal foam heat sinks fabricated with thermally sprayed interface," Applied Thermal Engineering, vol. 105, pp. 411424, Jul 252016. 